\title{
Impact of combined non-surgical and surgical periodontal treatment in patients with type 2 diabetes mellitus-a preliminary report randomized clinical study.
}

\author{
Dashnor Bukleta ${ }^{1,2}$, Shaip Krasniqi ${ }^{3}$, Giangiacomo Beretta $^{4}$, Armond Daci ${ }^{*}$, Arb Nila ${ }^{2}$, Teuta \\ Komoni $^{6}$, Manushaqe Selmani ${ }^{6}$, Brikene Elshani $^{7}$, Rok Schara $^{1}$ \\ ${ }^{1}$ Department of Oral Medicine and Periodontology, Faculty of Medicine, University of Ljubljana, Ljubljana, Slovenia \\ ${ }^{2}$ Department of Oral Surgery, Dental Polyclinic of Peja, Peja, Kosovo \\ ${ }^{3}$ Institute of Pharmacology Clinical Pharmacology and Toxicology, Faculty of Medicine, University of Prishtina, \\ Prishtina, Kosovo \\ ${ }^{4}$ Department of Pharmaceutical Sciences, Università Degli Studi di Milano, Milan, Italy \\ ${ }^{5}$ Department of Pharmacy, Faculty of the Medicine University of Prishtina, Prishtina, Kosovo \\ ${ }^{6}$ School of Dentistry, Faculty of Medicine, University of Prishtina, Prishtina, Kosovo \\ ${ }^{7}$ Faculty of Medicine, University of Prishtina, Prishtina, Kosovo
}

\begin{abstract}
Background/objectives: Scientific evidence regarding the effects of chronic periodontitis on Type 2 Diabetes Mellitus (T2M) is fragmentary and not definitive. This intervention study was designed to evaluate the effects of a Non-Surgical Procedure (NSP) in addition to a surgical procedure on systemic inflammation and glycaemic control in patients with T2M and periodontitis and Non-Diabetic (ND) patients with periodontitis.

Material and methods: A total of 100 patients with diabetes were randomly allocated to a treatment group and a control group. At least one tooth was extracted from each patient. After extraction, the control group $(n=50)$ did not receive any other treatment until the 3-month follow-up. Patients in the treatment group $(\mathrm{n}=50)$ received Full-Mouth Scaling and Root Planing (FM-SRP).The Mean Probing Depth (MPD), Mean Attachment Level (MAL), Plaque Index (PI), Bleeding on Probing (BOP), fasting blood samples for the measurement of high-sensitivity C-Reactive Protein (hs-CRP), and glycated hemoglobin (HbA1c) were measured or taken at baseline and 3 months after treatment. To investigate the effect of diabetes on the therapeutic procedure's outcome, ND patients with periodontitis $(n=60)$ were divided into two equal groups and subjected to the same procedures and analysis.

Results: HbA1c and hs-CRP decreased significantly in the diabetic groups and decreased more markedly when FM-SRP was added to tooth extraction $(P<0.001)$. For ND patients, all of the examined periodontal parameters were in favor of the combination therapy $(\mathbf{P}<0.001)$.

Conclusion: Non-surgical periodontal treatment can help improve the outcome of surgical periodontal procedures by reducing systemic inflammatory status and improve glucose metabolism.
\end{abstract}

Keywords: Diabetes mellitus type 2, Inflammation, Periodontitis, Diabetes, Periodontal treatment, Diabetes.

Accepted on November 14, 2017

\section{Introduction}

Type 2 Diabetes Mellitus (T2DM) is a chronic disease that comprises a heterogeneous group of metabolic disorders expressed with altered glucose tolerance or impaired carbohydrate metabolism. In diabetic patients with poorly controlled metabolism, complications develop more rapidly and in more severe forms. Systemic complications (retinopathy, nephropathy, neuropathy, macrovascular and microvascular disease) are associated with prolonged hyperglycemia $[1,2]$.

The prevalence of diabetes has drastically increased over the past decade, with economic conditions playing an important role due to poor medication adherence [3-5]. 
Currently, dental professionals play an important role in the process of diagnosing and monitoring DM [6]. A major role was shown to play also dental hygienists in the screening of patients with periodontitis [7].

It has been shown that DM is linked to various oral complications that include periodontal disease, which is classified as the sixth complication of diabetes. Studies have indicated that individuals with type $2 \mathrm{DM}$ are three times more vulnerable to developing periodontal oral complications than individuals without diabetes [8,9].

Moreover, periodontal disease is considered to be one of the major oral complications that are likely to occur in patients with poorly controlled type $2 \mathrm{DM}$ [10]. The prevalence of periodontal oral complications has been shown to increase with age. The loss of teeth or dental formulae is considered to be 15 times higher for individuals with DM than for those without DM [11]. A higher prevalence of periodontal disease is reported in individuals who rarely seek medical attention $[12,13]$.

Individuals with type 2 diabetes also exhibit poor oral health [14]. This implies, therefore, that glycaemic control is generally affected by oral complications such as periodontal infection and oral complications adversely affect periodontal health in individuals [15].

Similarly, periodontal disease increases the severity of DM and complicates glycaemic control. In general, improvements in glycaemic control improvements were shown to decrease the rate of acute and chronic systemic complications [16]. In addition, patients with well-controlled diabetes, as measured by the blood glycated hemoglobin levels, have the less severe periodontal disease than do individuals with poorly controlled diabetes [17]. The elimination of periodontal infection improves the glycaemic control of diabetes, as defined by a reduction in glycated hemoglobin. It was confirmed that even full-mouth tooth extraction raises the improvement magnitude [18].

It is known that inflammation plays a part in insulin resistance in patients with type 2 diabetes [19]. According to this finding, in periodontitis patients without other apparent diseases, CRP levels are higher than in patients without periodontitis [20]. Management of diabetic patients can maintain periodontal health with responding better to the periodontal treatment [21], and on the other side, the control of chronic periodontal infection is essential for achieving long-term control of T2DM [22].

Previous studies found that non-surgical periodontal therapy alone has an impact on the quality of life [23], and also such as full-mouth scaling and root planning resulted in an improvement of glycaemic control [24,25]. Moreover, another study demonstrated that non-surgical periodontal therapy was not proven to be effective in patients with T2DM with underlying obesity [26].

However, recent meta-analyses have suggested that this conclusion must be interpreted with care due to the limited robustness and heterogeneity of the studies available in the literature [27,28].

Taking this into consideration together with the necessity for further clinical trial investigations for T2DM and periodontal disease, the aim of the present study was to evaluate the effects of surgical treatment (tooth extraction) alone or in combination with non-surgical periodontal treatment (tooth cleaning) on periodontal status, systemic inflammation and glycaemic control in diabetic and non-diabetic patients with periodontal disease.

\section{Material and Methods}

\section{Study design}

In this study conducted during 2015-2016, based on a literature review, the data for 200 patients who were admitted to the endocrinology department of "Peja's Regional Hospital" and Dental Polyclinic in the city of Peja were assessed and examined for eligibility by oral surgery specialists. All procedures performed in our study involving human participants were in accordance with the ethical standards. The study was approved by the Ethics Committee of the University Clinical Centre of Kosovo (Nr.4212/2) and informed consent was obtained from all individual participants included in the study. A priori sample size calculation was performed given: Effect size $\mathrm{d}=0.5$, alpha error probability 0.08 and power 0.8 resulting in 26 patients for the group.

Personal interviews were used to collect baseline data from each participant using a pre-structured questionnaire. Patients were selected if they met the following inclusion criteria: had been diagnosed with type $2 \mathrm{DM}$; had a baseline $\mathrm{HbAlc} \geq$ $6.5 \%$, had at least 10 teeth in the functional dentition (excluding third molars); and had a clinical diagnosis of periodontal disease with at least one site with a Probing Depth (PD) $\geq 5 \mathrm{~mm}$, two teeth with attachment loss $\geq 6 \mathrm{~mm}$ and no modification in the pharmacological treatment of diabetes during the study period. Exclusion criteria included pregnancy or lactation, major diabetic complications, and the use of antibiotic therapy or non-steroidal anti-inflammatory drug therapy within 4 months before the first visit. Patient Characteristics and Baseline characteristics of biochemical and clinical data's from the patients are shown in (Tables 1 and 2).

After clinical examination, 160 patients aged $30-70$ y old were selected for this open-label, randomized clinical trial. These were further divided into four groups: type 2 DM groups (with or without initial periodontal therapy) and non-diabetic groups (with or without initial periodontal therapy) (Figure 1).

All the patients had periodontal disease, and at least one tooth extraction was performed for each patient. Prior to the surgical procedures, an adjunctive, non-surgical periodontal treatment to achieve a full-mouth tooth cleaning was performed for the patients in the treatment groups: Full-Mouth Scaling and Root Planing (FM-SRP) using an ultrasonic device (UDS-J Ultrasonic Scaler, Guilin Woodpecker Medical Instrument) and 
periodontal curettes for the mechanical debridement of supraand subgingival plaque and calculus.

Post-operative rinsing was followed by the use of the antiseptic solution Listerine ${ }^{\circledR}$ (ethanol $21.6 \%$, methyl salicylate $0.06 \%$, menthol $0.042 \%$, thymol $0.064 \%$ and eucalyptol $0.092 \%$ ) as a mouthwash thrice a day for $3 \mathrm{w}$. The study was registered on Clinical.Trials.gov (NCT02874963).

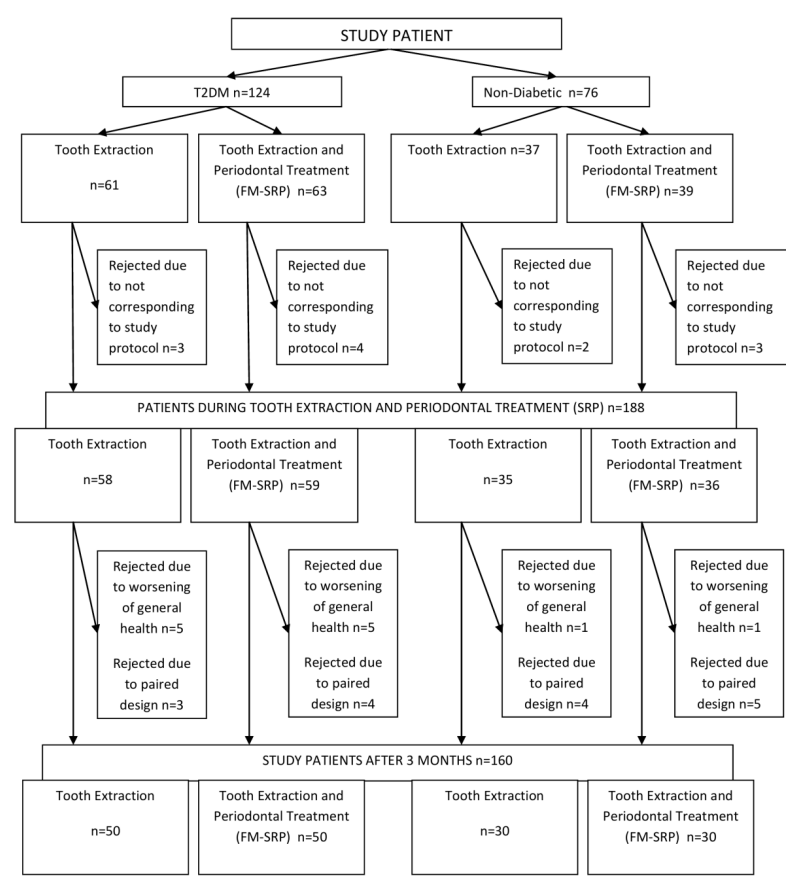

Figure 1. Study population flowchart.

\section{Clinical data collection}

The periodontal clinical examinations were performed according to the online periodontal chart (Department of Periodontology, School of Dental Medicine (ZMK), University of Bern) at baseline and 3 months after the periodontal treatment. All the parameters were recorded for six measurement points (mesial buccal, buccal, distal buccal, mesial lingual, lingual, distal lingual) on all teeth (excluding $3^{\text {rd }}$ molars) during each of two visits.

\section{Laboratory investigations}

The levels of glycosylated hemoglobin (HbAlc) and of highsensitivity C-reactive protein (hs-CRP) were measured using venous blood collected from patients at baseline and 3 months after the study procedures. All biochemical analyses were conducted in a biochemical laboratory in Peja (Laboratory Diagnostic Center, Peja, Kosovo) using a biochemistry analyzer (Select Pro XS, ELITech Clinical Systems, Paris, France) and enzyme-linked immunoassay kits (ELITech Clinical Systems, Paris, France).

\section{Statistical analyses}

All data were expressed as the means and Standard Deviations (SDs). Before the statistical analysis was performed, the normal distribution and homogeneity of the variances were tested. Associations between the experimental parameters were investigated using one-way ANOVAs, followed by t-tests of pairwise comparisons with the Least Square Difference (LSD) post hoc adjustment for multiple comparisons. Significant differences were also evaluated using the Mann-Whitney Utest, paired Student's t-test or the Wilcoxon rank-sum test. The results were considered significant when the $P$ value was $\mathrm{P}<0.05$ (GraphPad Prism 5.0 software).

\section{Results}

\section{Glycemic control and inflammation}

The FM-SRP treatment after tooth extraction-induced significant improvements in the serum HbA1c values in T2DM patients with periodontal disease.

No significant difference was found in the HbA1c plasma serum levels of ND patients $(5.56 \pm 0.62 \%$ vs. $5.65 \pm 0.65 \%$; $\mathrm{P}=0.099, \mathrm{n}=30$ ), (Figure 2A) or in ND patients treated with the combined surgical/non-surgical therapy $(5.79 \pm 2.92 \%$ vs. 5.58 $\pm 0.74 \%,(-3.6 \%) ; \mathrm{P}=0.036, \mathrm{n}=30)$ (Figure 2B).
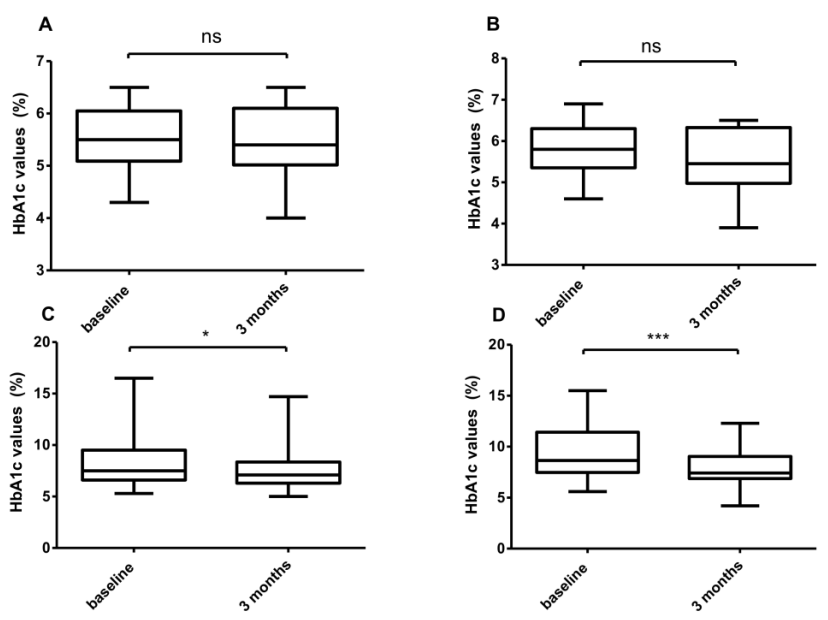

Figure 2. Graphical representation (box plot) of HbAlc values (\%) at baseline and 3 months after tooth extraction in (A) non-diabetic patients with periodontal disease, (B) non-diabetic patients with periodontal disease treated with FM-SRP, (C) T2DM patients with periodontal disease and (D) T2DM patients with periodontal disease treated with FM-SRP. ${ }^{*}$ indicates $p<0.05,{ }^{* *} p<0.01,{ }^{* * *} p<0.001, N S$ : Not Significant.

With regard to periodontal disease, only the T2DM patients showed a significant $\mathrm{HbA} 1 \mathrm{c}$ reduction after tooth extraction $(8.82 \pm 3.01 \%$ vs. $7.69 \pm 2.09 \%,(-13 \%) ; \mathrm{P}=0.018, \mathrm{n}=50)$, (Figure 2C) and after tooth extraction followed by tooth cleaning with FM-SRP $(9.59 \pm 2.57 \%$ vs. $8.03 \pm 1.67 \%$, $(-16.3 \%) ; \mathrm{P}=0.0003, \mathrm{n}=50)$ (Figure 2D). The variation in the serum levels of hs-CRP in ND patients was not statistically significant after tooth extraction $(1.64 \pm 1.06$ vs. $1.64 \pm 0.92$; 
$\mathrm{P}=0.913, \mathrm{n}=30$ ) (Figure 3A), but it was significant for patients with extracted teeth combined with FM-SRP $(3.63 \pm 2.19 \%$ vs. $3.11 \pm 1.95 \%,(-14 \%) ; \mathrm{P}=0.0006, \mathrm{n}=30)$ (Figure 3B). In T2DM patients with periodontal disease, the reduction of hs-CRP after treatment with either tooth extraction only or with tooth extraction combined with FM-SRP was significant and had a higher magnitude than that of the ND patients $(4.05 \pm 2.20 \mathrm{vs}$. $3.24 \pm 1.67,(-20 \%) ; \mathrm{P}=0.0003, \mathrm{n}=50)$ and $4.44 \pm 2.55$ vs. 2.85 $\pm 1.96,(-36.2 \%) ; \mathrm{P}=0.0009, \mathrm{n}=50$ ) (Figures $3 \mathrm{C}$ and $3 \mathrm{D}$, respectively).
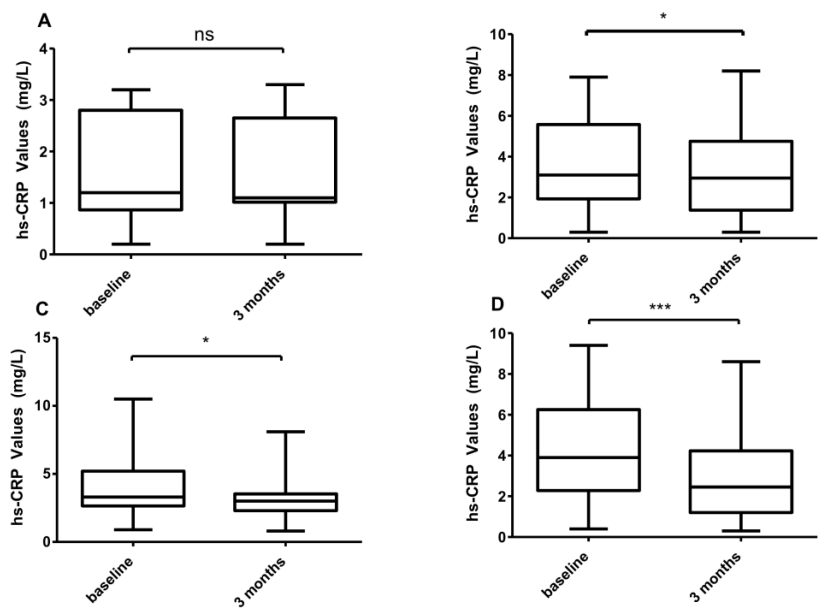

Figure 3. Graphical representation (box plot) of hs-CRP values $(\mathrm{mg} / \mathrm{L})$ at baseline and 3 months after tooth extraction in $(A)$ nondiabetic patients with periodontal disease, (B) non-diabetic patients with periodontal disease treated with FM-SRP, (C) T2DM patients with periodontal disease and (D) T2DM patients with periodontal disease treated with FM-SRP. ${ }^{*}$ indicates $p<0.05, \quad{ }^{* *} p<0.01$, ${ }^{* * *} p<0.001$.

\section{Periodontal conditions}

The MPD value decreased, although not significantly, in ND patients after tooth extraction without FM-SRP treatment, while the decrease observed in non-diabetic patients reached the level of statistical significance in the FM-SRP treated group. This variation was significant and slightly higher in both T2DM groups: extraction only $(6.60 \pm 1.47 \mathrm{~mm}$ vs. $5.24 \pm$ $2.17 \mathrm{~mm} ; \mathrm{P}=0.0004, \mathrm{n}=50)$ and extraction followed by $\mathrm{FM}$ $\mathrm{SRP}(6.52 \pm 0.82 \mathrm{~mm}$ vs. $5.08 \pm 1.10 \mathrm{~mm} ; \mathrm{P}=0.0001, \mathrm{n}=50)$ (Figure 4A). The MAL value decreased significantly only in T2DM patients after tooth extraction followed by FM-SRP $(-8.51 \pm 1.55$ vs. $-7.32 \pm 1.31 ; \mathrm{P}=0.0001, \mathrm{n}=50)$ (Figure 4B). Although the other groups showed a similar trend, those differences did not reach the level of statistical significance.

As expected, a significant reduction of PI was found in all groups with different magnitudes of reduction, depending on the FM-SRP application.

The lowest reductions were found in ND and T2DM patients after tooth extraction only $(95.07 \pm 7.14 \%$ vs. $86.23 \pm 12.51 \%$; $\mathrm{P}=0.0014, \mathrm{n}=30)$ and $(93.02 \pm 15.78 \%$ vs. $79.41 \pm 20.97 \%$; $\mathrm{P}=0.0001, \mathrm{n}=50$ respectively); the differences were enhanced by the FM-SRP treatment in both the ND and T2DM groups $(96.57 \pm 8.28$ vs. $61.07 \pm 25.27(\mathrm{n}=30) ; \mathrm{P}=0.0028$ and $98.06 \pm$
5.92 vs. $58.67 \pm 22.76 ; \mathrm{P}=0.0001, \mathrm{n}=50$ respectively) (Figure $5 \mathrm{~A})$.

BOP was reduced in all groups: ND patients after tooth extraction $(70.36 \pm 22.58 \%$ vs. $55.70 \pm 20.57 \% ; \mathrm{P}=0.011$, $\mathrm{n}=30) \mathrm{ND}$ patients after tooth extraction followed by FM-SRP (73.31 $\pm 32.26 \%$ vs. $48.57 \pm 29.72 \% ; \mathrm{P}=0.0031, \mathrm{n}=30), \mathrm{T} 2 \mathrm{DM}$ patients after tooth extraction only $(71.55 \pm 34.65 \%$ vs. $51.01 \pm$ $34.02 \% ; \mathrm{P}=0.0023, \mathrm{n}=50$ ), and $\mathrm{T} 2 \mathrm{DM}$ patients after tooth extraction in combination with FM-SRP $(87.76 \pm 23.89 \%$ vs. $28.94 \pm 21.65 \% ; \mathrm{P}=0.0001, \mathrm{n}=50$ ) (Figure 5B).

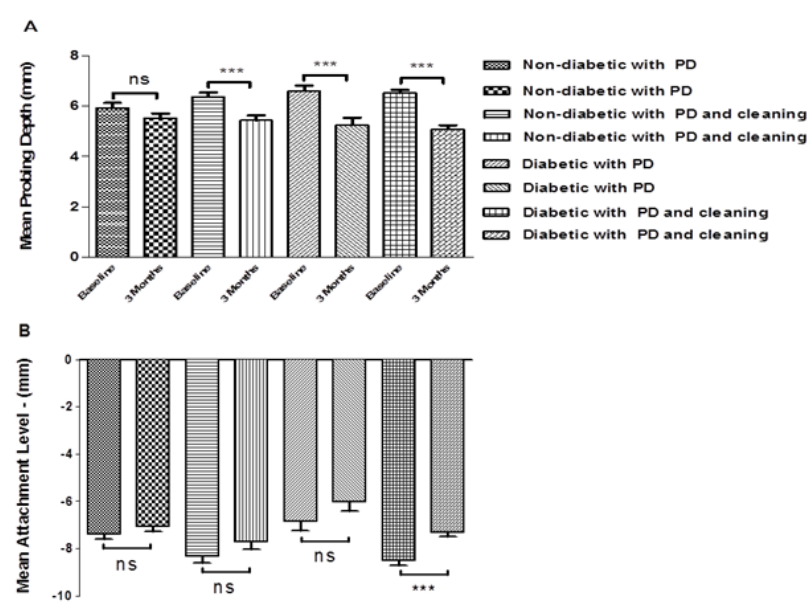

Figure 4. Comparison of mean probing depth ( $\mathrm{mm}$ ) (A) and mean attachment level $(\mathrm{mm})$ among groups $(B)$ with respect to time at baseline and 3 months after tooth extraction or extraction accompanied by periodontal treatment (FM-SRP). "indicates $p<0.05$, ${ }^{* *} p<0.01,{ }^{* * *} p<0.001$.

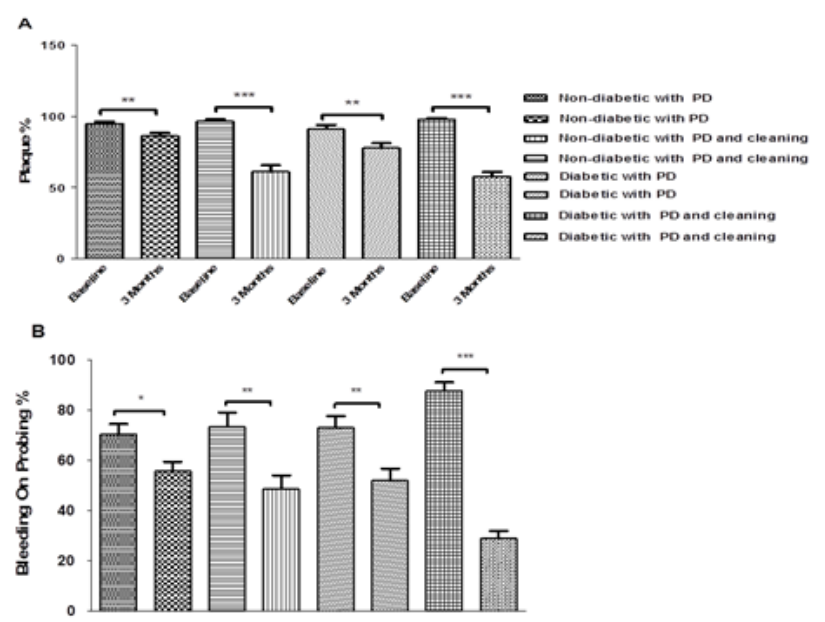

Figure 5. Comparison of plaque (\%) (A) and bleeding on probing (\%) (B) among groups with respect to time at baseline and 3 months after tooth extraction or extraction accompanied by periodontal treatment (FM-SRP). ${ }^{*}$ indicates $p<0.05,{ }^{* *} p<0.01,{ }^{* * *} p<0.001$.

Table 1. Patient characteristics.

\begin{tabular}{lll}
\hline Characteristics & T2DM & Controls \\
\hline Total patients $(n)$ & 100 & 60
\end{tabular}


Impact of combined non-surgical and surgical periodontal treatment in patients with type 2 diabetes mellitus-a preliminary report randomized clinical study

\begin{tabular}{lll}
\hline Sex (Male/female) & $50 / 50$ & $33 / 27$ \\
\hline Age $(\mathrm{y})$ & $59.49 \pm 10.82$ & $56.66 \pm 12.22$ \\
\hline Weight $(\mathrm{Kg})$ & $76.03 \pm 9.76$ & $78.3 \pm 9.76$ \\
\hline Height $(\mathrm{cm})$ & $174.64 \pm 13.96$ & $171.86 \pm 6.81$ \\
\hline
\end{tabular}

\begin{tabular}{lll}
\hline BMI & $26.01 \pm 3.64$ & $26.49 \pm 2.20$ \\
\hline No. of teeth extracted & $175(1.75)$ & $103(1.72)$ \\
\hline Oral therapy & 48 & - \\
\hline Insulin & 52 & - \\
\hline
\end{tabular}

Table 2. Baseline characteristics of biochemical and clinical data's from the patients.

\begin{tabular}{|c|c|c|c|c|c|}
\hline \multirow[t]{2}{*}{ Parameters } & \multicolumn{2}{|l|}{ Patients with diabetes } & \multicolumn{2}{|l|}{ Patients without diabetes } & \multirow{2}{*}{$\begin{array}{l}\text { Smokers } \\
\text { Diabetes/without diabetes }\end{array}$} \\
\hline & $\begin{array}{l}\text { Tooth extraction and } \\
\text { FM-SRP }\end{array}$ & Tooth extraction & $\begin{array}{l}\text { Tooth extraction and FM- } \\
\text { SRP }\end{array}$ & Tooth extraction & \\
\hline No. of teeth extracted & 87 & 88 & 54 & 49 & $88 / 37$ \\
\hline $\mathrm{PD}(\mathrm{mm})$ & $5.92 \pm 1.06$ & $6.37 \pm 0.93$ & $6.59 \pm 1.47$ & $6.53 \pm 0.83$ & $6.55 \pm 0.75 / 6.08 \pm 0.864$ \\
\hline $\mathrm{mAL}(\mathrm{mm})$ & $7.38 \pm 1.31$ & $6.8 \pm 1.68$ & $6.85 \pm 2.74$ & $8.52 \pm 1.56$ & $7.40 \pm 2.79 / 7.75 \pm 1.41$ \\
\hline $\mathrm{P}(\%)$ & $95.07 \pm 7.14$ & $96.57 \pm 8.28$ & $93.02 \pm 15.78$ & $98.06 \pm 5.92$ & $96.41 \pm 9.22 / 93.73 \pm 10.05$ \\
\hline BOP $(\%)$ & $70.36 \pm 22.58$ & $73.31 \pm 32.26$ & $71.55 \pm 34.65$ & $87.76 \pm 23.89$ & $79.57 \pm 30.99 / 72.74 \pm 27.33$ \\
\hline $\mathrm{HbA1c}(\%)$ & $8.82 \pm 3.01$ & $9.59 \pm 2.57$ & $5.56 \pm 0.62$ & $5.79 \pm 2.92$ & $8.98 \pm 2.48 / 5.76 \pm 0.63$ \\
\hline hs-CRP (mg/L) & $4.05 \pm 2.20$ & $4.44 \pm 2.55$ & $1.64 \pm 1.06$ & $3.63 \pm 2.19$ & $4.2 \pm 2.07 / 2.37 \pm 1.54$ \\
\hline
\end{tabular}

\section{Discussion}

The present study investigated the effects of surgical and nonsurgical treatments on the periodontal status, glycaemic control (HbAlc) and systemic inflammation (hs-CRP) of T2M and ND subjects with periodontal diseases. This study was conducted to detect the beneficial properties of full-mouth tooth cleaning after tooth extraction in T2DM patients with moderate to severe periodontitis.

The critical analysis of different periodontal parameters in the T2DM group and the control group enabled us to find valuable evidence that indicates that the study interventions are effective in reducing oral complications, particularly in T2DM patients.

Our results show that the all measured periodontal clinical parameters (MPD, MAL, BOP and PI) improved significantly in T2DM patients. These results are consistent with those from previous studies in which the effect of FM-SRP, subgingival curettage, and tooth cleaning treatments on glycaemic control has been evaluated [26,29-32].

Our results demonstrated that concomitant cleaning and tooth extraction is more beneficial than tooth extraction alone in the course of periodontal disease treatment for both non-diabetic and T2DM patients.

It is known that the progression of the periodontal disease as a chronic infection is a risk factor for the development of diabetes, and this was also observed in our study groups [33].

In addition, we used the HbAlc and hs-CRP levels as indicators of diabetic control and inflammation in both groups. Other studies have evaluated periodontal treatments such as topical antibiotics and surgical procedures and have shown significant improvements in $\mathrm{HbAlc}$ through the reduction of
hs-CRP levels, and these results are consistent with our findings $[31,34,35]$.

Similarly, following tooth extraction alone, patients tended to have improved HbAlc and hs-CRP values [17,36]. Oral hygiene plays an important factor in the control of periodontal status and in metabolic control [37].

It is well known that dental prophylaxis alone plays a role in periodontal health but does not affect the improvement of HbA1c levels [38].

Interestingly, in another study, full-mouth disinfection in type 1 diabetes patients has shown a positive effect on metabolic control [39]. In another meta-analysis, non-surgical periodontal treatment improved metabolic control in patients with periodontitis and diabetes [25]. Moreover, another recent study demonstrated that better oral hygiene improved periodontal health and metabolic control, which also confirms our findings [40].

These studies suggest a beneficial effect of surgical and nonsurgical treatments on glycaemic control and periodontal status. Hence, taking this into consideration, we confirm that tooth cleaning through FM-SRP increases the positive effect of tooth extraction in patients with the periodontal disease with or without T2DM, and we shed light on their importance in dental oral practice.

\section{Conclusion}

By showing the critical effects of tooth cleaning accompanied by tooth extraction in the response to and efficacy of treatment for periodontal disease patients with T2DM and without DM, our work may set the stage for larger investigational studies aimed at evaluating the impact of non-surgical and surgical 
approaches to the clinical management of periodontal disease and T2DM.

\section{Compliance and Ethical Standards}

\section{Conflict of interest}

The authors declare that they have no conflict of interest.

\section{Ethical approval}

"All procedures performed in our study involving human participants were in accordance with the ethical standards of the institutional and/or national research committee and with the 1964 Helsinki declaration and its later amendments or comparable ethical standards". The study was approved by the Ethics Committee of the University Clinical Centre of Kosovo (Nr.4212/2).

\section{Informed consent}

"Informed consent was obtained from all individual participants included in the study".

\section{Acknowledgment}

We would like to thank the Slovenian Human Resources Development and Scholarship Fund (SHRDSF) for the providing scholarship for Dr. Dashnor Bukleta.

\section{Authors' Contributions}

The study design was planned by $\mathrm{RC}, \mathrm{DB}, \mathrm{AD}$, and GB. Data collection was performed by DB, AN, TK, MS. Data analysis was conducted by RC, DB, AD, SK. All authors contributed to the interpretation of the data and manuscript preparation.

\section{References}

1. American Diabetes Association. Standards of medical care in diabetes-2014. Diab Care 2014; 37: 14-80.

2. Stratton IM, Adler AI, Neil HAW. Association of glycaemia with macrovascular and microvascular complications of type 2 diabetes (UKPDS 35): prospective observational study. BMJ 2000; 321: 405-412.

3. Zeqiri S, Ylli A, Zeqiri N. The effect of physical activity in glycemia in patients with diabetes mellitus. Med Arh 2006; 61: 146-149.

4. Jerliu N, Toçi E, Burazeri G, Ramadani N, Brand H. Prevalence and socioeconomic correlates of chronic morbidity among elderly people in Kosovo: a populationbased survey. BMC Geriatr 2013; 13: 1.

5. Daci A, Elshani B, Beretta G. gestational Diabetes Mellitus (gDM) in the republic of Kosovo: a retrospective pilot Study. Med Arh 2013; 67: 88.

6. Albert DA, Ward A, Allweiss P, Graves. Diabetes and oral disease: implications for health professionals. Ann N Y Acad Sci 2012; 1255: 1-15.
7. Bossart M, Calley KH, Guerenlian JR, Mason B, Ferguson RE. A pilot study of an HbAlc chairside screening protocol for diabetes in patients with chronic periodontitis: the dental hygiensts role. Int J Dent 2016; 14: 98-107.

8. Mealey BL, Oates TW. Diabetes mellitus and periodontal diseases. J Periodontol 2006; 77: 1289-303.

9. Loe H. Periodontal disease: the sixth complication of diabetes mellitus. Diab Care 1993; 16: 329-334.

10. Tsai C, Hayes C, Taylor GW. Glycemic control of type 2 diabetes and severe periodontal disease in the US adult population. Community Dent Oral Epidemiol 2002; 30: 182-192.

11. Touger-Decker R, Schaefer M, Flinton R, Steinberg L. Effect of tooth loss and dentures On diet habits. J Prosthet Dent 1996; 75: 831.

12. Kamberi B, Hoxha V, Stavileci M, Dragusha E, Kuçi A, Kqiku L. Prevalence of apical periodontitis and endodontic treatment in a Kosovar adult population. BMC Oral Health 2011; 11: 1 .

13. Begzati A, Meqa K, Siegenthaler D, Berisha M, Mautsch W. Dental health evaluation of children in Kosovo. Eur J Dent 2011; 5: 32-39.

14. Saremi A, Nelson RG, Tulloch-Reid M. Periodontal disease and mortality in type 2 diabetes. Diab Care 2005; 28: 27-32.

15. Borgnakke WS, Genco RJ. Periodontal disease and diabetes mellitus. International Textbook of Diabetes Mellitus (4th Edn.) 2015; 988-1004.

16. Norris SL, Engelgau MM, Narayan KV. Effectiveness of self-management training in type 2 diabetes a systematic review of randomized controlled trials. Diab Care 2001; 24: 561-587.

17. Soskolne WA, Klinger A. The relationship between periodontal diseases and diabetes: an overview. Ann Periodontol 2001; 6: 91-98.

18. Khader YS, Al Habashneh R, Al Malalheh M, Bataineh A. The effect of full-mouth tooth extraction on glycemic control among patients with type 2 diabetes requiring extraction of all remaining teeth: a randomized clinical trial. J Periodontal Res 2010; 45: 741-747.

19. Nesto R. C-reactive protein, its role in inflammation, type 2 diabetes and cardiovascular disease, and the effects of insulin-sensitizing treatment with thiazolidinediones. Diabet Med 2004; 21: 810-817.

20. Paraskevas S, Huizinga JD, Loos BG. A systematic review and meta-analyses on C-reactive protein in relation to periodontitis. J Clin Periodontol 2008; 35: 277-290.

21. AlJehani YA. Risk factors of periodontal disease: review of the literature. Int J Dent 2014.

22. Grossi SG, Genco RJ. Periodontal disease and diabetes mellitus: a two-way relationship. Ann Periodontol 1998; 3: 51-61.

23. Goel K, Baral Dh. A comparison of impact of chronic periodontal diseases and nonsurgical periodontal therapy on oral health-related quality of life. Int J Dent 2017. 
preliminary report randomized clinical study

24. Corbella S, Francetti L, Taschieri S, Siena F, Fabbro MD. Effect of periodontal treatment on glycemic control of patients with diabetes: A systematic review and metaanalysis. J Diabetes Investig 2013; 4: 502-509.

25. Kıran M, Arpak N, Unsal E, Erdoğan MF. The effect of improved periodontal health on metabolic control in type 2 diabetes mellitus. J Clin Periodontol 2005; 32: 266-272.

26. Kapellas K, Mejia G, Bartold PM, Skilton MR, MapleBrown LJ. Periodontal therapy and glycaemic control among individuals with type 2 diabetes: reflections from the Periocardio study. Int J Dent 2016.

27. Teeuw WJ, Gerdes VE, Loos BG. Effect of periodontal treatment on glycemic control of diabetic patients a systematic review and meta-analysis. Diab Care 2010; 33: 421-427.

28. Wang X, Han X, Guo X, Luo X, Wang D. The effect of periodontal treatment on hemoglobin alc levels of diabetic patients: a systematic review and meta-analysis. PLoS One 2014; 9: 108412.

29. Altamash M, Klinge B, Engström PE. Periodontal treatment and $\mathrm{HbAlc}$ levels in subjects with diabetes mellitus. J Oral Rehabil 2016; 43: 31-38.

30. Rodrigues DC, Taba M, Novaes AB, Souza SL, Grisi MF. Effect of non-surgical periodontal therapy on glycemic control in patients with type 2 diabetes mellitus. J Periodontol 2003; 74: 1361-1367.

31. OConnell PA, Taba M, Nomizo A. Effects of periodontal therapy on glycemic control and inflammatory markers. J Periodontol 2008; 79: 774-783.

32. Chen L, Luo G, Xuan D, Wei B, Liu F, Li J, Zhang J. Effects of non-surgical periodontal treatment on clinical response, serum inflammatory parameters, and metabolic control in patients with type 2 diabetes: a randomized study. J Periodontol 2012; 83: 435-443.

33. Demmer RT, Desvarieux M, Holtfreter B, Jacobs DR, Wallaschofski H, Nauck M, Völzke H, Kocher T. Periodontal status and A1C change longitudinal results from the study of health in pomerania (SHIP). Diab Care 2010; 33: 1037-1043.
34. Katagiri S, Nitta H, Nagasawa T. Multi-center intervention study on glycohemoglobin (HbAlc) and serum, highsensitivity CRP (hs-CRP) after local anti-infectious periodontal treatment in type 2 diabetic patients with periodontal disease. Diabetes Res Clin Pract 2009; 83: 308-315.

35. Sun WL, Chen LL, Zhang SZ, Wu YM, Ren YZ, Qin GM. Inflammatory cytokines, adiponectin, insulin resistance and metabolic control after periodontal intervention in patients with type 2 diabetes and chronic periodontitis. Intern Med 2011; 50: 1569-1574.

36. Taylor BA, Tofler GH, Carey HM. Full-mouth tooth extraction lowers systemic inflammatory and thrombotic markers of cardiovascular risk. J Dent Res 2006; 85: 74-78.

37. Sandberg GE, Sundberg HE, Fjellstrom CA, Wikblad KF. Type 2 diabetes and oral health: a comparison between diabetic and non-diabetic subjects. Diabetes Res Clin Pract 2000; 50: 27.

38. Lopez NJ, Quintero A, Casanova PA, Martinez B. Routine prophylaxes improves chronic periodontitis status in type 2 diabetes. J Periodontol 2014; 85: 232-240.

39. Schara R, Medvescek M, Skaleric U. Periodontal disease and diabetes metabolic control: a full-mouth disinfection approach. J Int Acad Periodontol 2006; 8: 61-66.

40. Pranckeviciene A, Siudikiene J, Ostrauskas R, Machiulskiene V. Long-term effect of periodontal surgery on oral health and metabolic control of diabetics. Clin Oral Investig 2016; 1-9.

\section{*Correspondence to}

Armond Daci

Department of Pharmacy

Faculty of Medicine

University of Prishtina

Kosovo 